\title{
Editorial
}

\section{Big data and the internet of things}

Journal of Marketing Analytics (2015) 3, 1-4. doi:10.1057/jma.2015.7

\section{ANALYTICS ON THE RISE}

We are entering the era of Big Data - the unmistakable realization that the current abundance and availability of unprecedented amounts of data will have a lasting impact on the way we run businesses.

McKinsey talks about Big Data as the fourth business revolution. They picture autonomous computerized manufacturing with processes that are interconnected by the Internet of Things (IoT). This promise of efficient, flexible, and customizable production relies heavily on Big Data: analytical systems that ingest very large volumes of data.

The first industrial revolution began with the introduction of steam-powered machines. Next came mass production, and then electronics and IT. This fourth industrial revolution, Big Data, holds the promise of yet another step change in productivity.

In manufacturing, we envision processes that govern themselves by relying on auto corrective actions to avoid waste and trigger timely replenishment of resources. This allows factories to schedule process downtime intelligently after detection of process deviations that signal the need for preventive maintenance or repair.

The IoT holds tremendous potential to change industrial manufacturing. But other industries will be affected, too. Uber is a great example of a new and innovative business that uses location technology and smartphone capabilities. When a customer needs a ride, drivers in the vicinity get notified, arrival time, ride fare and duration are predicted, all based on the optimal route which depends on the time of day. Examples abound of new business models like Uber, in various industries, that rely on Big Data.
Many parties contribute to the hype around Big Data. Storage providers, IT solution providers that focus on NoSQL, NewSQL, and consultancy companies all stand to gain a lot by the surge in demand for talent and resources to fuel this wave. These commercial parties are often held responsible for 'hype creation', advertising the latest and greatest to help drive their business growth.

In all fairness, it were not the commercial parties that initiated awareness for Big Data. Over the past 10 years, we have seen an array of business books pointing to the rise of, for want of a better description, data driven decision making. Large-scale application of this new technology perforce calls for data gathering efforts of ever increasing scope. Big Data just keeps getting bigger.

As with many new trends, it is hard to point to the 'true' origin. Some of the publications that have sold in large numbers, and that have emphasized the value of analytics, were books like: 'Competing on Analytics' (Davenport \& Harris, 2007), 'Super Crunchers' (Ayres, 2008), 'Data Driven' (Redman, 2008), 'The Numerati' (Baker, 2008), 'Numbers Rule your World' (Kaiser Fung, 2010), 'The Signal and the Noise' (Silver, 2012), 'Predictive Analytics' (Siegel, 2013) and several others.

In my opinion, the current notion of Big Data is not so radically 'new' at all. Data warehousing, as a discipline, was invented in the 90's. Big Data providers refer to 'data lakes', but to me this architectural concept looks strikingly similar to either a persistent staging area (Kimball et al, 2008) or an operational data store (Inmon, 1999), which are data warehouse components that are well-established, and have been in use for two decades. 
The term 'data warehouse' suggests persistent storage of data, as well as temporal modeling of data. Reality is that many practical implementations have to put limitations on persistence because of storage costs and performance issues (query response times). All this makes the supposedly new concept of data lakes look even more like already existing data warehouses. Data modeling needs to happen eventually, either centrally and more upfront, or by data users (decentralized and implicit) and downstream of storage. Again, not so radically different.

The current Big Data hype does seem to make this trend more tangible to a wider audience. Add some marketing sauce to it, and you begin to understand why everybody is jumping on this bandwagon. After all: if even McKinsey considers it a big deal, well, then there really must be something there, right?

Nobody stores data for the sake of storage. The payoff comes from meaningful insights. 'Analytics' is the output of Big Data that can create value in many ways. When data point to changes in market dynamics, or sliding customer preferences, this provides an opportunity to act. In this context, analyst firm Gartner refers to 'Pattern based strategies'.

Gartner points out that companies already compete on analytics, for instance, they use data to determine which market segments to go after, and which ones they are happy to ignore (do not consider commercially viable). If companies can discern these trends in the marketplace only a little bit sooner or better than their competitors, they will enjoy a constant edge over the market.

Big Data is relevant to executives because it provides an opportunity to gain competitive advantage. Shortages in energy, materials, food and even water put a limit on unrestricted growth. Innovation needs to come from doing more with less. This is where analytics come in. If analytics then also leverage proprietary data (to which competitors cannot get access), this becomes a sustainable source of competitive advantage.

The first analytics wave was fueled by data that arose from man-machine interactions.
This second, IoT wave of analytics, is spurred by machine-machine interactions that give rise to unprecedented volumes of data. Leveraging the IoT in Big Data applications is indeed new, and offers potential for genuine business innovation.

\section{THE INTERNET OF THINGS, A TSUNAMI COMING IN}

As if the hype around Big Data wasn't enough, the next data explosion is already coming up: the IoT. IoT refers to non-human internet traffic, as opposed to humans triggering exchange of data via the internet protocol.

'Things' in IoT are devices and machines that themselves are either directly used by people (say, an ATM), or that people rely on, like traffic signaling systems and so on. These devices or machines exchange internet messages ('traffic') among each other. It's this machine-to-machine data that is now making Big Data so much bigger.

The idea of leveraging machine-to-machine data exchange in support of customer needs is not entirely new. It is claimed that as far back as 1982 Carnegie Mellon University had a soda machine connected to the internet so that users could monitor availability and temperature of drinks remotely.

This still limits applicability somewhat, because one end of the communication chain is constrained by human bandwidth.

Someone has to look up if their favorite drink is in stock and properly chilled. This implies that the amount of information flow is practically constrained by human attention. People have limited capacity to process information and therefore growth of these types of solutions is bounded.

However, beginning somewhere in the 90's, device-to-device communication removed one end of that constraint (the human side), which opened the door to almost unlimited growth of data exchange. The concurrent rise of RFID technology played a part in that development as well. 
The introduction of barcodes and scanners to keep track of SKU's had a similar effect in the retail industry, albeit at a somewhat smaller scale. This in and of itself has led to remarkable data growth. Not just storage of raw data, but also by embedding solutions in logistics systems. To get a sense of scale, an analytical system like Walwart's data warehouse boasts over 500 Terabytes of data.

Gartner says there will be 5 billion connected devices in 2015, and that number will grow to 25 billion by 2020 . Many new technologies rely on the internet for data exchange. Mobile internet is (almost) ubiquitous in the western world. Wide availability of wifi and increasing internet bandwidth (for example: fiber optics, DSL and so on) have made this architecture a viable approach.

Telemetry will increasingly be recorded via the web. In many cases, these sensors (telemetrics) are leveraging the new IPv6 protocol for their data interchange. This is why Vinton Cerf (Google's internet guru) has predicted that demand for IPv6 addresses will skyrocket.

To get a sense of this uncanny explosion in data volumes, a transatlantic flight generates about three Terabytes of raw data, and those data are just from sensors on board. Google's self-driving car is said to generate about 750 $\mathrm{Mb} / \mathrm{sec}$. That is just the raw data, making this data available for analysis (for instance, to analyze traits of safe drivers) requires additional transformations, which would make it even bigger.

This second wave of Big Data growth, triggered by large-scale application of machine-to-machine traffic, is more like a tsunami than a wave. Unstoppable and irreversible. Recording and storing these data has become economically viable. Among others, NoSQL solutions have dramatically reduced costs. The cloud business model has plummeted storage prices, and computing power still keeps improving in accordance with Moore's law, even after half a century.

A graphic calculator that kids use in high school is now about six times more powerful that the computer on board of Apollo 11 that put Neil Armstrong and Buzz Aldrin on the moon. In the 60 's, a famous prediction was made that eventually about a dozen Cray super computers would suffice to satisfy the computing needs for the entire nation. A Sony Playstation 4 game computer today is more powerful. So much for predictions...

All of this technology has secured a place in our everyday lives. Most of these applications go largely unnoticed; you would almost take them for granted. As we introduce new solutions to improve resource efficiencies and generally better our lives, even more possible ways to leverage all these data will be explored.

\section{BIG DATA AND THE IOT ARE CHANGING THE PLAYING FIELD}

We can harness Big Data systems and employ them to create customer value. Application of advanced analytics is the cornerstone of this fourth business revolution. Predictive modeling, smart adaptive systems, and self-governing processes are examples how this may come to bear. Robotics and artificial intelligence (AI) are coming of age, ever smarter systems enter the mainstream of our lives.

Deep Blue, IBM's most powerful AI computer first beat Kasparov at chess in 1997 and in 2011 IBM's Watson (a natural language AI system) won Jeopardy. This proves that AI can now successfully 'compete' with humans on information processing tasks. What was dramatic progress yesterday becomes established technology tomorrow.

As an example, until the 1970's people (underwriters) would assess credit requests, until it became clear that computer algorithms had become more apt at making these judgments. Initially this ran counter to intuition because underwriting is such a knowledge intensive task. Statistical models seem overly simplistic by comparison. However, the data (monitoring of accepted 
accounts over time) showed that computers accepted fewer bad creditors than people did, no matter how hard they tried.

The result of this finding was a change in competitive dynamics. To avoid credit losses and stay in business, one company after the other had little choice but to adopt the (initially counterintuitive) process of application credit scoring by statistical models. For a few decades now, this has become standard practice.

So many of these mainstream applications add to the growth in data volumes, and increasingly that exchange will also leverage the internet protocol. All of this will contribute to even more data growth. On a small scale, a simple devices like an electronic car key (when the car has a start button instead of a key slot) are constantly interacting with the vehicle. If you drive off (after starting the car) without the key, the interruption in communication between key and car gets noticed, and your dashboard will signal accordingly. If you try to stop the engine, the car will remind you again that the key is absent before you are able to shut it off.

On a larger scale, smart meters help homeowners save on their heating and electricity bill. Utility providers benefit by being able to forecast demand, and create value for their customers by extending recommendations. The data interchange between millions of smart meters and the smart power grid will help reduce energy consumption and create efficiencies for utility providers - a win-win.

Some things this overview has aimed to point out:

1. Although Big Data is an important phenomenon, it isn't nearly as 'new' as the hype seems to suggest; with broader application of the IoT, Big Data offers new and even bigger opportunities.

2. The human imagination consistently falls short in dreaming up how the future might look different from the past. Future applications of analytics will prove no different.

In this issue of the Journal of Marketing Analytics we have two articles on Big Data. One deals with ethics, because amassing all of these data has many implications for privacy and ethics. Another article explains how Big Data can be combined or merged with data from consumer panels, another largely unexplored area of value from Big Data. A third article deals with decision-making frameworks, a model for leveraging the output from Analytics. These are all timely topics, and reflect contemporary developments in our professional world.

Machine-to-machine interactions are bound to lead to new technological and consumer applications. Innovations that we can only dream of - if our imagination were rich enough. As new applications enter the mainstream of our daily lives, and technology matures, even more ways to leverage the IoT are bound to break through.

\section{REFERENCES}

Ayres, I. (2008) Super Crunchers, New York, NY: Bantam Books.

Baker, S. (2008) The Numerati, New York, NY: Houghton Mifflin Harcourt.

Davenport, T. and Harris, J (2007) Competing on Analytics: The New Science of Winning, Boston, MA: Harvard Business School Press.

Inmon, W.H. (1999) Building the Operational Data Store, 2nd edn. New York, NY: Wiley.

Kaiser Fung (2010) Numbers Rule your World: The Hidden Influence of Probabilities and Statistics on Everything You Do, New York, NY: McGraw-Hill Education.

Kimball, R., Ross, M., Thornthwaite, W., Mundy, J. and Becker, B. (2008) Data warehouse Lifecycle Toolkit, 2nd edn. New York, NY: Wiley.

Redman, T. (2008) Data Driven: Profiting from Your Most Important Business Asset, Boston, MA: Harvard Business School Publishing.

Siegel, E. (2013) Predictive Analytics: The Power to Predict Who Will Click, Buy, Lie, or Die, New York, NY: Wiley.

Silver, N. (2012) The Signal and the Noise: Why So Many Predictions Fail - but Some Don't, New York, NY: Penguin Books.

Tom Breur

E-mail: tom.breur@cengage.com 